\title{
Multiple UAVs Nonlinear Guidance Laws for Stationary Target Obser- vation with Waypoint Incidence Angle Constraint
}

\author{
Mingu Kim* and Youdan Kim** \\ School of Mechanical and Aerospace Engineering, Seoul National University, Seoul 151-742, Republic of Korea.
}

\begin{abstract}
Nonlinear guidance law combined with a pseudo pursuit guidance is proposed, to perform stationary target observation mission. Multiple UAVs are considered, with waypoint constraint. The whole guidance is divided into two steps: firstly, waypoint approach, with specified incidence angle; and secondly, loitering around the stationary target. Geometric approach is used to consider the constraint on the waypoint, and a specified phase angle between the loitering UAV and the approaching UAV. In the waypoint approach step, UAVs fly to the waypoint using the pseudo pursuit guidance law. After passing the waypoint, UAVs turn around the target, using a distance error dynamics-based guidance law. Numerical simulations are performed, to verify the performance of the proposed guidance law.
\end{abstract}

Key words: Nonlinear guidance law, Target observation, Virtual waypoint, Pursuit guidance law

\section{Introduction}

Recently, many studies on autonomous flights of Unmanned Aerial Vehicles (UAVs) have been performed, since UAVs have been used to accomplish various missions. In particular, target observation mission has been widely studied, because it has very important applications, such as aerial reconnaissance and surveillance. Multiple small UAVs may substitute for a large UAV, for performing complicated missions, due to their low cost and easy operation, and therefore various guidance laws for multiple UAVs have been developed [1-8].

A vector field approach has been studied for standoff tracking missions of multiple UAVs [1-6]. A Lyapunov vector field was used to calculate the desired velocity and heading rate of UAVs, for performing coordinate standoff tracking of a stationary or moving target [1-4]. Chen et al. modified the Lyapunov vector field, by combining a tangent vector field, to find a short path for a UAV tracking a ground target [5]. Lawrence et al. extended the Lyapunov vector field guidance for path and waypoint passing, by switching circular loiter vector fields [6]. The Lyapunov vector field guidance law guarantees global stability, by showing limit cycle behavior about circular loiter patterns. However, due to the limit cycle behavior, it is difficult to implement it in missions in complexly constrained environments.

Distance error dynamics were also utilized, to design a guidance law for performing stationary target observation $[7,8]$. Distance error and Line-of-Sight (LOS) information were used to guide a UAV to the observation circle, utilizing the characteristics of distance error dynamics. Distance error dynamics-based guidance law has a simple structure, but it does not guarantee the satisfaction of various constraints on time or angle.

This paper deals with a waypoint constraint problem on the observation circle. Time and angle constraints to approach the desired circle path could become important issues for multiple UAVs operation, to avoid them colliding with each other, and to perform the mission effectively. While the time and angle constraints problem has not been treated much in UAV systems, similar problems have been widely studied in missile systems [9-12]. Impact time and impact angle constraints in missile systems, which have the same meaning of approach time and incidence angle, are very important in
This is an Open Access article distributed under the terms of the Creative Commons Attribution Non-Commercial License (http://creativecommons.org/licenses/by$\mathrm{nc} / 3.0 /$ which permits unrestricted non-commercial use, distribution, and reproduction in any medium, provided the original work is properly cited.
* * Graduate Student
** Professor, Corresponding author: ydkim@snu.ac.kr 
attacking a target. For this reason, a nonlinear guidance law used in missile systems is considered, to solve the waypoint constraint problem in this study.

Pseudo pursuit guidance law and distance error dynamicsbased guidance law are utilized, to perform the stationary target observation of multiple UAVs, with waypoint incidence angle constraint. The whole guidance logic consists of two steps: the waypoint approach step, with specified incidence angle; and the loitering step, around the stationary target. In the first step, UAVs fly to the waypoint, using pseudo pursuit guidance law. After passing the waypoint, UAVs turn around the target, using the distance error dynamics-based guidance law, which is the second step.

The purpose of the paper is to propose a nonlinear guidance scheme, by implementing two nonlinear guidance laws that are easy to understand and simple to implement. Therefore, the proposed guidance scheme is used to satisfy the waypoint incidence angle constraint, and to perform the stationary target observation mission.

This paper is organized as follows. Section 2 provides the waypoint determination, using the geometric relation among UAVs and the stationary target. Pseudo pursuit guidance law considering the desired heading angle and distance error dynamics-based guidance law is also described. Section 3 shows numerical simulations for verifying the performance of the proposed nonlinear guidance scheme. Finally, the conclusion is given in Section 4.

\section{Problem Statement}

\subsection{Waypoint Determination}

Waypoint constraint could be given, to complete a given mission effectively and successfully. In this study, the following assumptions are considered. First, the velocity of the leader UAV is constant, but the velocity of the follower UAV could be changed, to maintain the distance from another UAV. Second, in the beginning, all UAVs fly to their initial waypoint. However, when the leader UAV passes its waypoint, and starts to loiter around the stationary target, the follower UAVs' desired waypoints are changed, according to the position of the leader UAV turning around the target. Third, follower UAVs have the information of the leader UAV position and turning direction. The first and second assumptions are the mission considered in this study, and the third one is necessary for UAVs to avoid colliding with each other. Figure 1 describes the waypoint determination process of the follower UAVs, while the leader UAV turns around the stationary target.

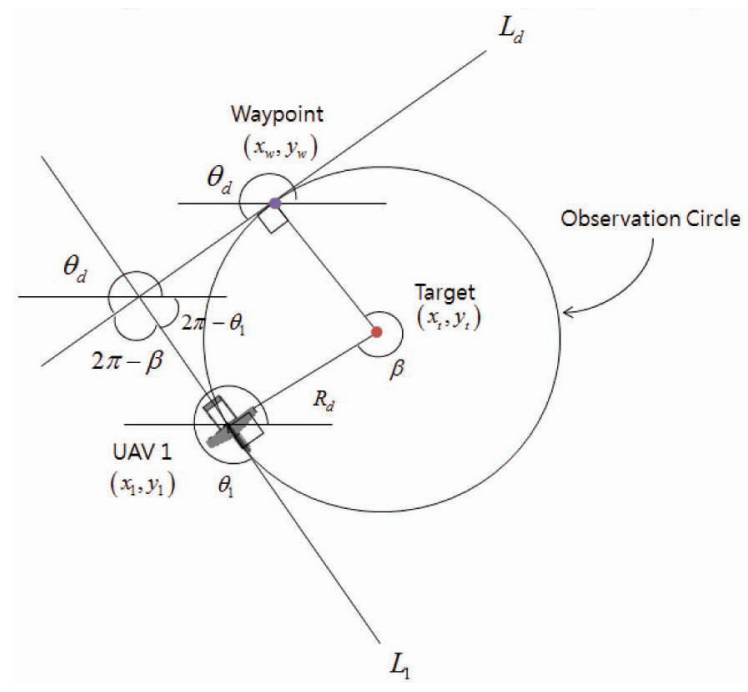

Fig. 1. Waypoint Determination

In Fig. $1,\left(x_{1}, y_{1}\right),\left(x_{t}, y_{t}\right)$, and $\left(x_{w}, y_{w}\right)$ are the positions of the leader UAV loitering around the stationary target, the stationary target, and the waypoint of the follower UAV on the observation circle, respectively. $R_{d}$ is the desired observation radius of the observation circle, $L_{1}$ and $L_{d}$ are the tangent line of the leader UAV turning around the stationary target, and tangent line of the follower UAV approaching the waypoint, respectively. $\theta_{1}, \theta_{d}$, and $\beta$ are the heading angle of the leader UAV, the desired heading angle of the follower UAV approaching the waypoint, and the phase angle difference between UAVs, respectively.

Definition 1. The directions of UAV LOS angle and UAV heading angle are positive counterclockwise (CCW) from the $\mathrm{x}$-axis. The direction of the phase angle is positive CCW from the radius of the loitering UAV.

From the geometry in Fig. 1, the desired heading angle of the approaching UAV can be obtained as

$$
\theta_{d}=\theta_{1}+\beta-2 \pi
$$

where, $\beta \in[-\pi, \pi]$.

For the operation of multiple UAVs, the turning direction of each UAV should correspond with the turning direction of other UAVs, to avoid colliding with each other. To determine the waypoint that the follower UAV approaches on the observation circle, the desired LOS angle should be obtained.

Proposition 1. For a CW turn case, the desired LOS angle is determined by subtracting $\pi / 2$ from the desired heading angle. On the other hand, for a CCW turn case, the desired LOS angle is obtained by adding $\pi / 2$ to the desired heading angle. 


$$
\left\{\begin{array}{lc}
\sigma_{d}=\theta_{d}-\frac{\pi}{2} & C W \\
\sigma_{d}=\theta_{d}+\frac{\pi}{2} & C C W
\end{array},\right.
$$

where, $\sigma_{d}$ is the desired LOS angle. Figure 2 shows the geometric relation between the desired heading angle, and the desired LOS angle, for both cases.

Then, the position of the approaching waypoint on the observation circle is calculated as

$$
\begin{aligned}
& x_{w}=x_{t}-R_{d} \cos \sigma_{d} \\
& y_{w}=y_{t}-R_{d} \sin \sigma_{d}
\end{aligned},
$$

The approaching waypoint will move along the observation circle whose radius is $R_{d}$, as the leader UAV loiters around the target. The movement rate of the waypoint can be obtained, by differentiating equation (3) as

$$
\begin{aligned}
& \dot{x}_{w}=R_{d} \dot{\sigma}_{d} \sin \sigma_{d} \\
& \dot{y}_{w}=-R_{d} \dot{\sigma}_{d} \cos \sigma_{d},
\end{aligned}
$$

Let us substitute eqs.(2) and (3) to eq.(4).

$$
\begin{aligned}
& \dot{x}_{w}=R_{d} \dot{\theta}_{1} \sin \left(\theta_{1}+\beta-\frac{3}{2} \pi\right) \\
& \dot{y}_{w}=-R_{d} \dot{\theta}_{1} \cos \left(\theta_{1}+\beta-\frac{3}{2} \pi\right),
\end{aligned}
$$

Finally, the movement rate of the waypoint can be represented as

$$
\begin{aligned}
& \dot{x}_{w}=R_{d} \dot{\theta}_{1} \cos \left(\theta_{1}+\beta\right) \\
& \dot{y}_{w}=-R_{d} \dot{\theta}_{1} \sin \left(\theta_{1}+\beta\right)
\end{aligned}
$$

As the approaching waypoint of the follower UAV moves, the follower UAV should accelerate or decelerate, to reduce or increase the phase angle between the leader UAV and the follower UAV, and finally maintain the desired phase angle difference. Therefore, the following velocity command is required for the follower $\operatorname{UAV}[1,2]$.

$$
\mathrm{V}_{c m d}=k\left(\theta_{1}-\theta_{d}\right) R_{d}+\mathrm{V}_{0},
$$

where, $k$ is the velocity command gain, and $\mathrm{V}_{0}$ is the nominal velocity of the UAV.

\subsection{Pseudo Pursuit Guidance Law}

Pursuit guidance is one of the widely used guidance laws for a missile system. Pseudo pursuit guidance law was introduced for automatic landing of a fixed wing UAV to a recovery net [13]. While the pure pursuit guidance law leads a UAV to a true target directly, the pseudo pursuit guidance law generates a guidance command for the vehicle to a virtual target. As the virtual target moves to the true target, the UAV finally arrives at the true target. Figure 3 explains the concept of the pseudo pursuit guidance law.

In Fig. $3, L$ is a distance between the UAV and the virtual target, and $\eta$ is the angle between the velocity vector of the UAV and the virtual target. It is assumed that the acceleration command is perpendicular to the velocity vector of the UAV in the pseudo pursuit guidance law. The acceleration command can be calculated as

$$
a_{c}=2 \frac{\mathrm{V}_{M}^{2}}{L} \sin \eta
$$

To satisfy the waypoint constraint, the virtual target is sequentially generated on the tangent line, $L_{d}$, and the virtual target moves to the true waypoint along the line.

Figure 4 shows the flight path of the UAV, and the movement of the virtual target on the tangent line, by using
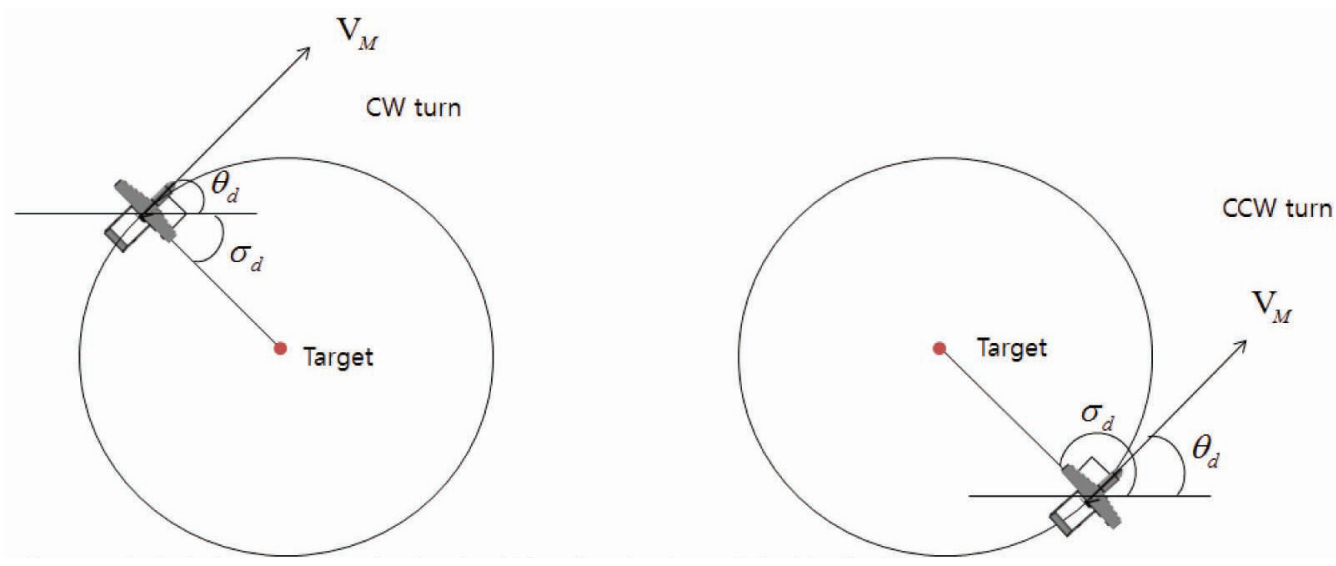

Fig. 2. The Geometric Relation between the Desired Heading Angle and the Desired LOS angle 
the pseudo pursuit guidance law.

Let us assume that the flight direction of the UAV is the same as the velocity vector direction of the UAV. In other words, the heading of a UAV aligns with its velocity vector. Then, the angle between the velocity vector and the virtual target, $\eta$, can be calculated as

$$
\eta=\theta_{M}-\tan ^{-1}\left(\frac{y_{i}-y}{x_{i}-x}\right),
$$

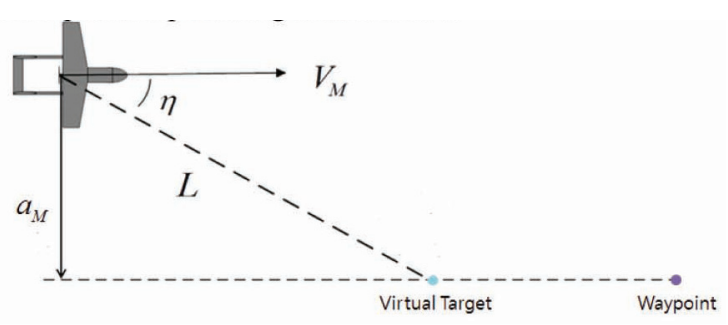

Fig. 3. The Concept of Pseudo Pursuit Guidance Law

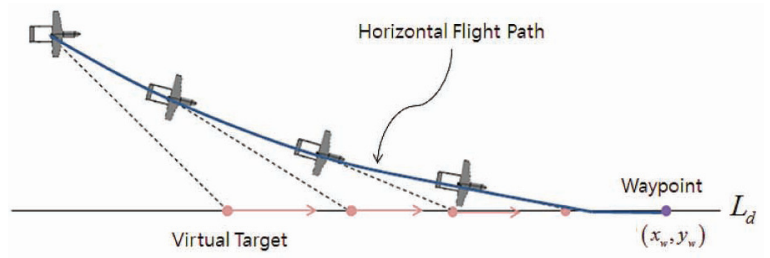

Fig. 4. The Generated Horizontal Flight Path, using Pseudo Pursuit Guidance Law

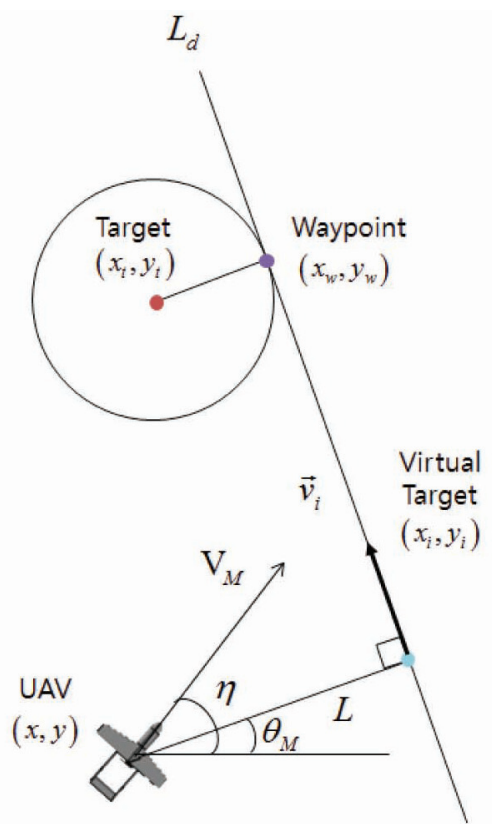

Fig. 5. The Geometric Relation between the UAV and the Imaginary Target where, $\theta_{M}$ is the heading angle of the UAV, $\left(x_{i}, y_{i}\right)$ is the position of the virtual target, and $(x, y)$ is the position of the UAV. Figure 5 shows the geometric relation between the heading angle of the UAV, and the LOS angle to the virtual target. In Fig. $5, \vec{v}_{i}$ represents the direction vector of the virtual target movement on the tangent line.

Now, let us consider another important issue, that is, how to determine the virtual target. Note that the imaginary target is located on the tangent line, and it moves to the waypoint. Therefore, the following equation can be obtained from the geometric relation between the virtual target and the true waypoint.

$$
\vec{L}_{d} \times \vec{v}_{i}=0
$$

Let us assume that the direction vector of the virtual target is perpendicular to the line between the UAV and the virtual target. Then, the following equation can be obtained.

$$
\vec{L} \bullet \vec{v}_{i}=0,
$$

Let us redefine the temporary virtual target, satisfying the above assumption, and the geometric relation between the true waypoint and the virtual target. Then, the position of the temporary virtual target can be calculated as

$$
\begin{aligned}
& x_{i t}=c_{1}^{2} x+c_{1} s_{1}\left(y-y_{w}\right)+s_{1}^{2} x_{w} \\
& y_{i t}=-\frac{c_{1}}{s_{1}}\left(x_{i t}-x\right)+y
\end{aligned}
$$

where, $c_{1}=\cos \theta_{d}$, and $s_{1}=\sin \theta_{d} \neq 0$.

If the desired heading angle is set as 0 or 180 degrees, then the position of the temporary virtual target should be chosen as

$$
\begin{aligned}
& x_{i t}=x \\
& y_{i t}=y_{w}
\end{aligned},
$$

To rapidly guide the UAV, the virtual target should be located in front of the temporary virtual target, considering the direction of the movement. Figure 6 shows the geometric relation of the true waypoint, the virtual target, and the temporary virtual target.

In Fig. 6, $L_{t}$ is the distance between the UAV and the temporary virtual target, $L_{i t}$ is the distance between the virtual target and the temporary virtual target, and $L_{w t}$ is the distance between the true waypoint and the temporary virtual target, respectively. Finally, the position of the virtual target can be obtained as

$$
\begin{aligned}
& x_{i}=x_{i t}+L_{i t} c_{1} \\
& y_{i}=y_{i t}+L_{i t} s_{1}
\end{aligned},
$$




\subsection{Distance Error Dynamics-Based Guidance Law}

After passing the waypoint, the guidance system will be changed from the pseudo pursuit guidance law, to the distance error dynamics-based guidance law. In this section, the distance error dynamics-based guidance law is briefly summarized $[7,8]$. Consider a point mass UAV model, as shown in Fig. 7. The following assumptions are required for the distance error dynamics-based guidance law. First, the autopilot and sensor dynamics of the UAV are significantly faster than the UAV dynamics. Second, the velocity of the UAV is constant during loitering. Third, the angle-of- attack (AOA) of the UAV is very small, and may be neglected. Finally, the acceleration vector of the UAV is perpendicular to the velocity vector of the UAV. Then, the equations of motion can be represented as

$$
\begin{aligned}
& \dot{R}=-\mathrm{V}_{M} \cos \left(\sigma-\theta_{M}\right), \\
& \dot{\sigma}=\frac{\mathrm{V}_{M}}{R} \sin \left(\sigma-\theta_{M}\right), \\
& \dot{\theta}_{M}=a_{M} / \mathrm{V}_{M}=a_{c_{n}},
\end{aligned}
$$

where, $R$ is the distance between the UAV and the target, $V_{M}$ is the velocity, $a_{M}$ is the acceleration, and $\sigma$ is the line-of-sight to the target. The distance error is defined as

$$
e_{R}=R-R_{d},
$$

Differentiating eq. (18) with respect to time, once and twice, yields

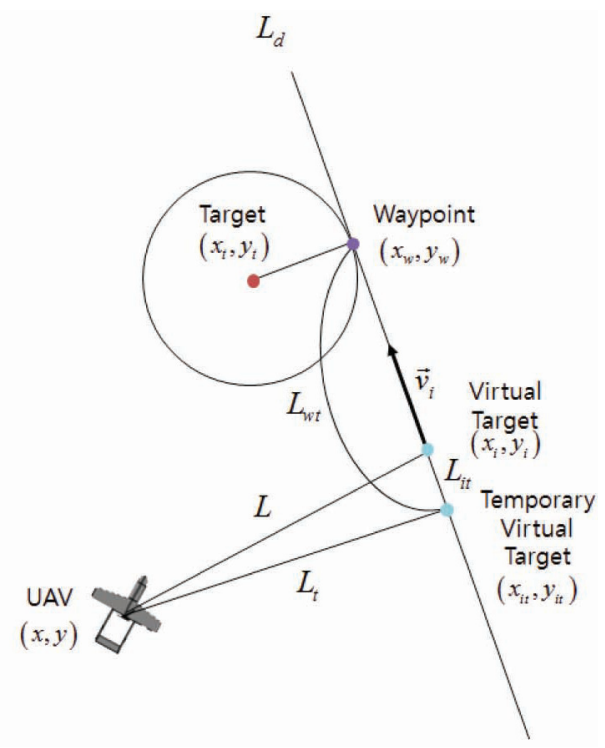

Fig. 6. The Geometry of Virtual Target

$$
\begin{aligned}
& \dot{e}_{R}=\dot{R}, \\
& \ddot{e}_{R}=\ddot{R},
\end{aligned}
$$

Using eqs. (15)-(17), the second derivative term of the distance error, eq. (20), can be rewritten as

$$
\begin{aligned}
\ddot{R} & =\mathrm{V}_{\mathrm{M}} \sin \left(\sigma-\theta_{\mathrm{M}}\right)\left(\dot{\sigma}-\dot{\theta}_{\mathrm{M}}\right), \\
& =R \dot{\sigma}^{2}-R \dot{\sigma} a_{c_{n}}
\end{aligned}
$$

Substituting eq. (20) into eq. (21), we have

$$
\ddot{e}_{R}-R \dot{\sigma}^{2}+R \dot{\sigma} a_{c_{n}}=0,
$$

Let us propose a normalized centrifugal acceleration command $a_{c_{n}}$ as

$$
a_{c_{n}}=\frac{1}{R \dot{\sigma}}\left(k_{1} \dot{e}_{R}+k_{2} e_{R}+R \dot{\sigma}^{2}\right),
$$

Then, substituting eq. (23) into eq. (22), the distance error dynamics can be represented as

$$
\ddot{e}_{R}+k_{1} \dot{e}_{R}+k_{2} e_{R}=0,
$$

Two guidance gains, $k_{1}$ and $k_{2}$, can be determined using various methods, such as the pole selection method, oscillation motion method, and Lyapunovbased gain selection method $[7,8]$. Note that the first derivative term of the LOS angle to the target is in the denominator of the guidance command, in eq. (22). This means the error dynamics-based guidance law is inadequate for the tracking problem of a straight path. However, in this study, the distance error dynamicsbased guidance law is used to rotate around the target, after passing the waypoint. Therefore, the acceleration command will be constant; and there is no straight path for tracking, when the distance error-based guidance law is used.

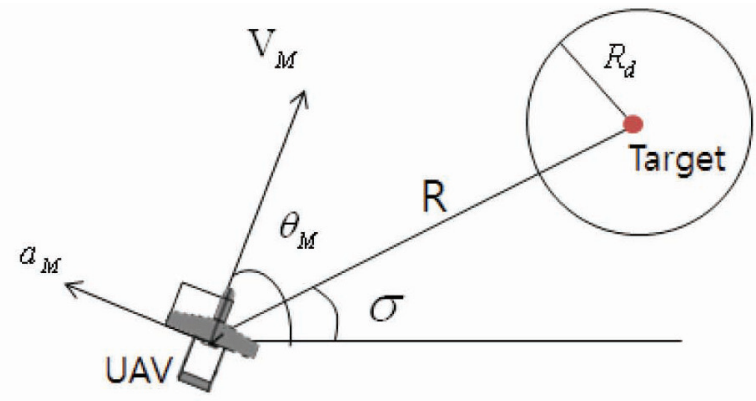

Fig. 7. Point Mass Model Geometry 


\section{Numerical Simulation}

To verify the performance of the proposed guidance law, numerical simulation is performed. The considered mission is the coordinated stationary target observation of multiple UAVs, with the waypoint constraint. The waypoints of the follower UAVs move on the desired observation circle, after the leader UAV passes through its waypoint.

In the numerical simulation, the nominal velocity of each $\mathrm{UAV}$ is $14 \mathrm{~m} / \mathrm{s}$, and the desired observation radius is chosen as $300 \mathrm{~m}$. The initial position of the leader $\operatorname{UAV}$ is $(0,0)$, and the positions of the follower UAVs are $(1800,0)$ and $(-100$, $1600)$, respectively. The target position is $(800,600)$. The limit of guidance command is chosen as $2 g / \mathrm{V}_{M}$, where $g$ is the gravitational acceleration. The velocity command gain of the follower UAVs is set as 0.002 , and the limit of the velocity command is set as $18 \mathrm{~m} / \mathrm{s}$. The distance error dynamicsbased guidance gains are chosen by the Lyapunov-based gain selection method [8]. In this study, the following positive semi-definite Lyapunov candidate function is considered.

$$
f_{L}=c_{1}\left(\frac{\mathrm{V}_{M}}{R}\right)^{2} e_{R}^{2}+c_{2} \dot{R}^{2} \geq 0,
$$

Then, the normalized centrifugal acceleration command of eq. (23) can be rewritten as

$$
a_{c_{n}}=\frac{1}{c_{2} R \dot{\sigma}}\left(c_{1} \mathrm{~V}_{M}^{2} \frac{e_{r} R_{d}}{R^{3}}+c_{2} R \dot{\sigma}^{2}+c_{3} \frac{\mathrm{V}_{M}}{R} \dot{R}\right)
$$

Finally, the first derivative of the Lyapunov candidate function is negative semi-definite.

$$
\dot{f}_{L}=-2 c_{3} \frac{\mathrm{V}_{M}}{R} \dot{R}^{2} \leq 0,
$$

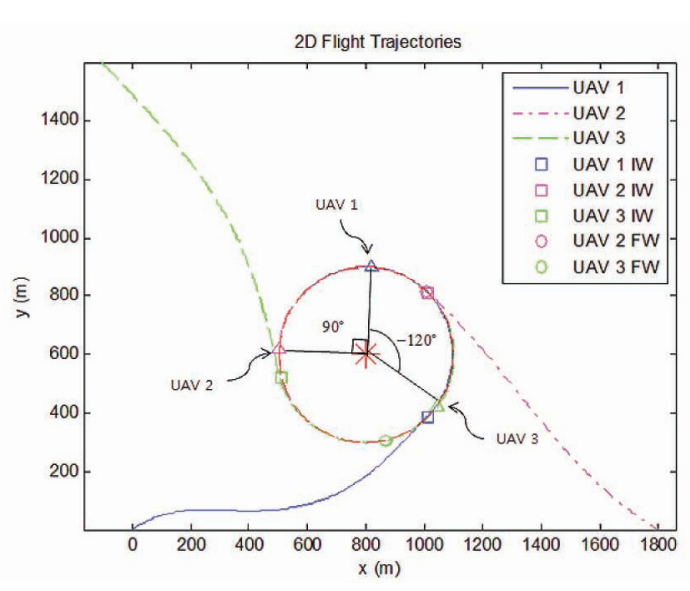

Fig. 8. 2-D Flight Trajectories in CCW turn
The proposed Lyapunov candidate function is a continuously differentiable function, such that for some $r>0$, $\Omega_{r}=\left\{\mathbf{x} \in \mathbb{R}^{n} \mid f_{L}<r\right\}$ is bounded, and its first derivative is negative semi-definite, where $\mathbf{x}=\left\{e_{R}, \dot{R}\right\}$. Let $D$ be the set of all points within $\Omega_{r}$ where $\dot{f}_{L}$.

$$
D=\left\{\mathbf{x} \in \mathbb{R}^{2} \mid \dot{R}=0\right\},
$$

and $M$ is the largest invariant set in $D$.

$$
M=\left\{\mathbf{x} \in \mathbb{R}^{2} \mid e_{R}=0, \dot{R}=0\right\},
$$

If $M$ contains a point with $e_{R} \neq 0$, then

$$
\ddot{R}=-\frac{c_{1}}{c_{2}} \frac{\mathrm{V}_{M}^{2} e_{R} R_{d}}{R^{3}}-\frac{c_{3}}{c_{2}} \frac{\mathrm{V}_{M}}{R} \dot{R} \neq 0,
$$

Remember that $c_{1}, c_{2}$, and $c_{3}$ are positive constants. Therefore, the trajectory will immediately move out of $D$, and thus out of $M$. This is in contradiction to the definition of $M$. Thus, according to LaSalle's invariance theorem, the normalized centrifugal acceleration command makes the UAV system be asymptotically stable. In the numerical simulation, $c_{1}, c_{2}$, and $c_{3}$, are set as 2,1 , and 3 , respectively.

Figure 8 shows the 2-dimensional flight trajectories of three UAVs, turning around the target counter clockwise. In the CCW turn case, the considered phase angle 1 between the leader UAV (UAV1) and the first follower UAV (UAV 2 ) is set as 90 degrees, and the phase angle 2 between the leader UAV and the second follower UAV (UAV 3) is chosen as -120 degrees. The initial waypoints (IW) of the three UAVs are represented as square dots in Fig. 8. Once the leader UAV (UAV 1) has passed through its waypoint, the follower UAVs' waypoints move along the desired observation circle, as shown in Fig. 8. The final waypoints (FW) of the follower
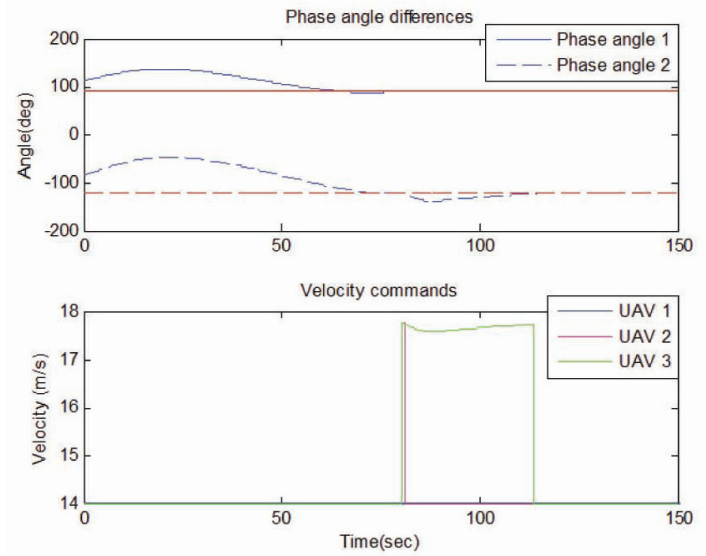

Fig. 9. Time History of Phase Angle Differences and Velocity Com mands in CCW turn 
UAVs are described as circular dots. Triangular dots are the positions of three UAVs loitering around the given target at 130 seconds. As shown in Fig. 8, the phase angle differences are maintained.

Figure 9 shows the time history of the phase angle differences and the velocity commands, in the CCW turn case. After all UAVs have passed their waypoints, the phase angles among UAVs maintain the desired values, as shown in Fig. 9. Also, the velocity commands are generated to pass the corresponding waypoint, for maintaining the desired phase angle differences, as shown in Fig. 9.

Figure 10 shows the time history of normalized guidance commands in the CCW turn case. In the simulation, the limit of the normalized centrifugal acceleration guidance command is chosen as about $1.4 s^{-1}$. As shown in Fig. 10, each normalized guidance command is below the specified limit.

In the CW turn case, the initial conditions, guidance gains, the nominal velocity of each UAV, and the limit of
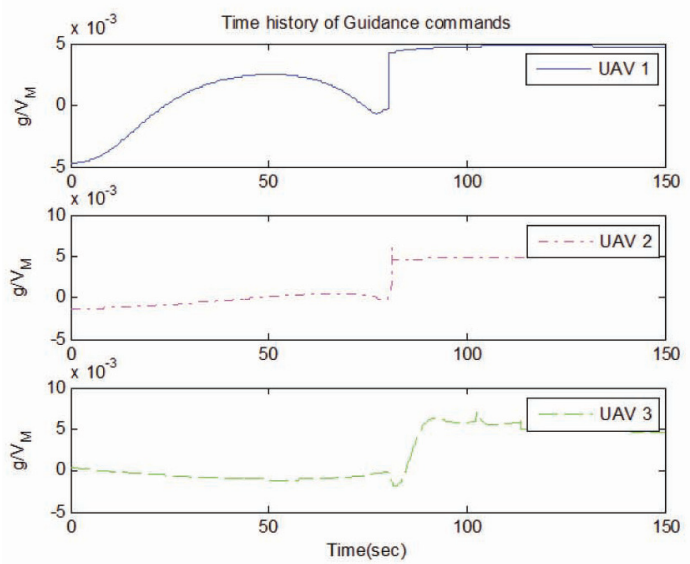

Fig. 10. Time History of Normalized Acceleration Guidance Commands in CCW turn

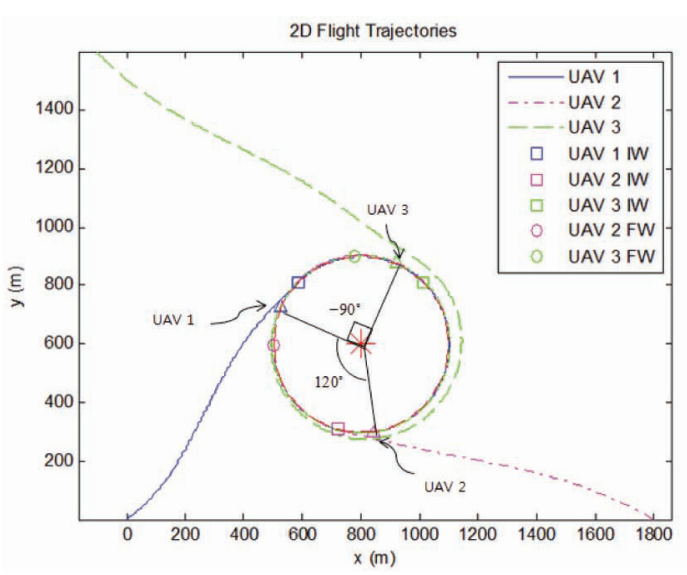

Fig. 11. 2-D Flight Trajectories in CW turn the guidance command and the velocity command are the same as in the CCW turn case. And, the considered phase angle 1 between the leader UAV (UAV1) and the first follower UAV (UAV 2) is set as 120 degrees, and the phase angle 2 between the leader UAV and the second follower UAV (UAV 3) is chosen as -90 degrees. Figure 11 shows the 2-dimensional flight trajectories of three UAVs turning around the target clockwise. The triangular dots are the positions of three UAVs loitering around the given target at 200 seconds.

Figure 12 shows the time history of the phase angle differences and the velocity commands, in the CW turn case. Again, the phase angle difference is maintained, as shown in Figs. 11 and 12. Figure 13 shows the time history of the normalized acceleration guidance commands in the CW turn case. As shown in Fig. 13, each normalized acceleration guidance command is below the limit of the normalized guidance command.
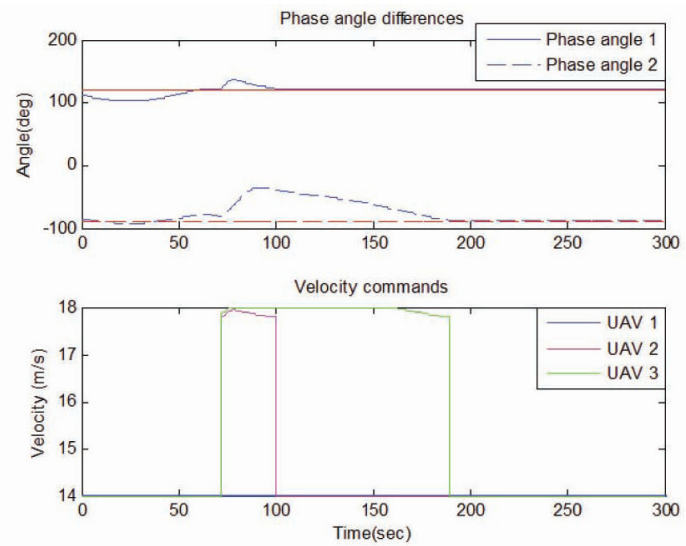

Fig. 12. Time History of Phase Angle Differences and Velocity Commands in CW turn
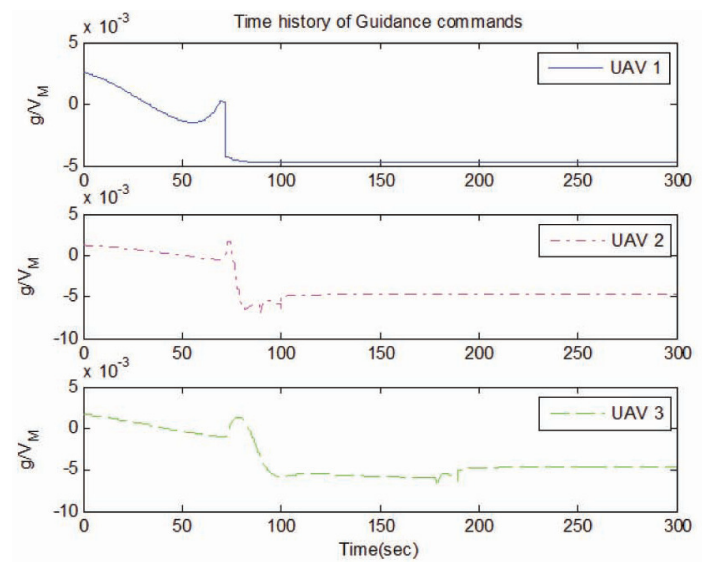

Fig. 13. Time History of Normalized Acceleration Guidance Commands in CW turn 


\section{Conclusion}

Pseudo pursuit guidance law and distance error dynamicsbased guidance law are combined, to perform stationary target observation, considering the waypoint constraint of multiple UAVs. Pseudo pursuit guidance law is used, to satisfy the waypoint constraint. After a UAV has passed through its waypoint on the observation circle, distance error dynamics-based guidance law is used, to loiter around the stationary target. To avoid colliding with the leader UAV, and improve the mission capability, the phase angle difference is considered to determine the waypoints of follower UAVs, and pseudo pursuit guidance law is analyzed geometrically, to guide the UAV to the waypoint. Numerical simulation is performed, to verify the performance of the proposed nonlinear guidance scheme, in the clockwise and counter clockwise turn cases. The proposed guidance law can be used to perform surveillance or fire monitoring mission of small multiple UAVs.

\section{Acknowledgement}

This work was supported by a Defense Research Grant, funded by the Agency for Defense Development, under the contract UD120013JD.

\section{References}

[1] Elston, J., and Frew, E. W., "Net-Centric Cooperative Tracking of Moving Targets," AIAA Infotech@Aerospace 2007 Conference and Exhibit, Rohnert Park, CA, 2007.

[2] Frew, E. W., Lawrence, D. A., and Morris, S., "Coordinated Standoff Tracking of Moving Targets Using Lyapunov Guidance Vector Fields," Journal of Guidance, Control, and Dynamics, Vol. 31, No. 2, 2008, pp. 290-306.

[3] Summers, T. H., Akella, M. R., and Mears, M. J., "Coordinated Standoff Tracking of Moving Targets: Control Laws and Information Architectures," Journal of Guidance,
Control, and Dynamics, Vol. 32, No. 1, 2009, pp. 56-69.

[4] Zhu, S., Wang, D., and Chen, Q., "Standoff Tracking Control of Moving Target in Unknown Wind," 48th IEEE Conference on Decision and Control, Shanghai, China, 2009.

[5] Chen, H., Chang, K. C., and Agate, C. S., "Tracking with UAV using Tangent-plus-Lyapunov Vector Field Guidance," 12th International conference on Information Fusion, Seattle, WA, 2009.

[6] Lawrence, D. A., Frew, E. W., and Pisano, W. J., "Lyapunov Vector Fields for Autonomous UA Flight Control," Journal of Guidance, Control, and Dynamics, Vol. 31, No. 5, 2008, pp. 1220-1229.

[7] Kim, M., Kim, Y., and Park, C. G., "Guidance Gain Analysis via Oscillation Motion of Error DynamicsBased Guidance Law for Stationary Target Observation," 4th European Conference for Aero-Space Sciences, St. Petersburg, Russia, 2011.

[8] Kim, M., and Kim, Y., "Error Dynamics-Based Lyapunov Guidance Law for Stationary Target Observation," 18th IFAC World Congress, Milan, Italy, 2011.

[9] Kim, K.-S., Kim, Y., "Design of Generalized Conceptual Guidance Law using Aim Angle," Control Engineering Practice, Vol. 12, No. 3, 2004, pp, 291-298.

[10] Ryoo, C.-K., Cho, H., and Tahk, M.-J., "Optimal Guidance Laws with Terminal Impact Angle Constraint," Journal of Guidance, Control, and Dynamics, Vol. 28, No. 4, 2005, pp. 724-732.

[11] Lee, J.-I., Jeon, I.-S., and Tahk, M.-J., "Guidance Law to Control Impact Time and Angle," IEEE Transactions on Aerospace and Electronic Systems, Vol. 43, No. 1, 2007, pp. 301-310.

[12] Zhou, D., Sun, S., and Teo, K. L., "Guidance Laws with Finite Time Convergence," Journal of Guidance, Control, and Dynamics, Vol. 32, No. 6, 2009, pp. 1838-1846.

[13] Yoon, S., Kim, H. J., and Kim, Y., "Spiral Landing Guidance Law Design for Unmanned Aerial Vehicle NetRecovery," Journal of Aerospace Engineering, Proceedings of the Institution of Mechanical Engineers Part G, Vol. 224, No. 10, 2010, pp. 1081-1096. 